\title{
The Study of the Digital Clothing Virtual Showcase and Network Customize Business System
}

\author{
Hu shouzhong, Gao changkuan, Hu hongyan \\ China Shanghai University of Engineering Science \\ hushzh@sues.edu.cn, 172012241@qq.com, 452521294qq.com
}

\begin{abstract}
With the development of digital and information technology, the position of E-commerce in the retail formats is increasingly important. This article studies the core technology of public E-commerce platform in clothing industry chain, including personalized virtual display system for three-dimensional body model generation system, clothing dynamic presentation and virtual online dressing system functional modules. Finally, this article discusses the application of the business system, which aims at giving the public platform of the E-commerce marketing in garment industry chain a support and promoting the practical application and commercialization of the digital clothing in Yangtze River Delta region.

Index Terms - Digital clothing. Virtual showcase. Network customize
\end{abstract}

\section{I . Introduction}

With the development of digital and information technology, the position of E-commerce in the retail formats is increasingly important. Now, from many shopping network which in domestic or abroad market, clothing mostly shows itself in the form of photo. However, clothing is a special commodity, which emphasizes the affinity of apparel merchandise and the body of consumers. Generally it will affect consumers' purchasing psychology if there is no effect of the dressing process. Simultaneously, the apparel "three-dimensional system" launched worldwide is mostly clothing threedimensional display system which can be seen the mode dressing effect of the" analog ". But the physical characteristics of the consumers "real" three-dimensional fitting, customized network custom clothing apparel Ecommerce development is the key of R \& D.

This study follows the "12th Five-Year Development Plan" of Shanghai textile and garment industry, launching research cooperation with large-scale backbone enterprises owned by the Shanghai Textile Holding (Group) Company, which makes joint efforts to develop the core technology of public E-commerce platform for the clothing industry chain, including personalized virtual display system for threedimensional human body model generation system, clothing dynamic presentation and virtual online fitting system functional modules. And all of them provide support for the building of the E-commerce marketing public platform for garment industry chain in Shanghai (and the Yangtze River Delta region), which aimed to promote the practical application of digital clothing and commercial operation ( see Fig.1).

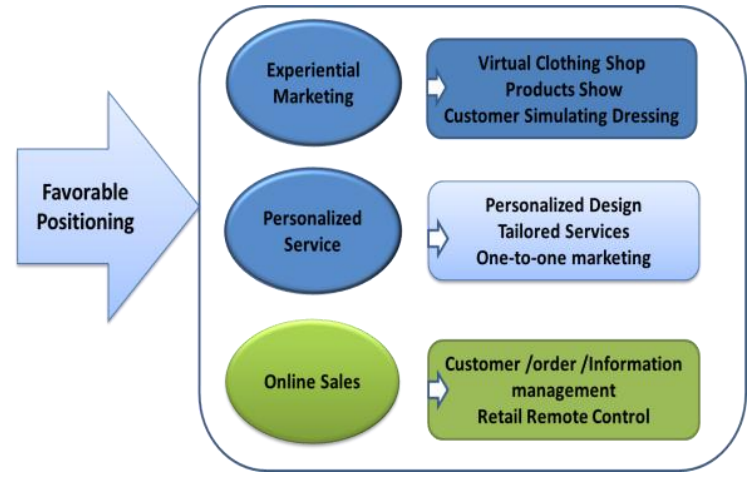

Fig. 1 the advantages positioning of E-MARKETING

II. Apparel network virtual exhibitions, the core module for customization and the technology roadmap

A. The deficiencies in the commerce platform of apparel network marketing system

Currently, the problems exist in clothing network customization and commerce virtual platforms mainly reflect in:

1) the obstacles appear in sense of reality of the network retail environment order and the experience of dressing. Lacking the function of virtual dressing and feeling the cloth texture features, which is the mainly psychological barriers for consumers to buy clothes and the most urgent problem need to be addressed between businesses and consumers in B2C Ecommerce;

2) the support for the clothing virtual exhibitions and digitized customized technology is imperfect in the clothing virtual network environment. The performance of the fabrics and the wearing effect of the "reality" are not digitized virtual, also the online customized clothing and apparel model system is not complete, which makes the custom clothing digitized sales environment not be achieved.

$B$. Apparel network virtual exhibitions, the core module for customization and the technology roadmap

The "clothing customization and virtual experience" core function module in the digitized apparel e-commerce system, which includes personalized three-dimensional human body model generated system, clothing dynamic presentation virtual display system, virtual online dressing, digitized apparel customized system .All of them aim to develop the clothing ecommerce retail and trading platform which meets the digital technology requirements of (see Fig. 2). 


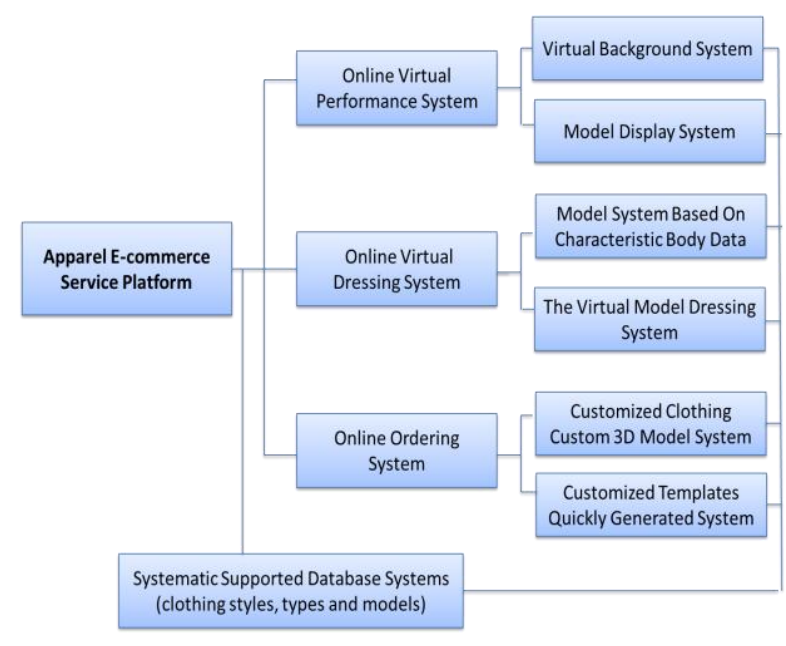

Fig. 2 Apparel network virtual exhibitions, the core module for customization and the technology roadmap

1) Apparel virtual online dressing system - the threedimensional virtual fitting system which is quick, easy and realistic: utilize three-dimensional clothing model which based on the method of the apparel entities rotating body depends on the reconstruction, to realize virtual wearing through matching the corresponding control points and surfaces (curvature) in the 3D model of body - clothing and spline template-based 3D elastic deformation algorithms;

2) Clothing dynamic virtual display system: the dynamic clothing virtual display system, which support the product release and demonstration of the network virtual, the system can generate clothing and dynamically modify the apparel attributes and parameters. Also supply the Fashion show scene editor that generates T-station, three-dimensional architectural scene and natural landscape, which demonstrates the clothing effect by using the game engine technology. The key problems need to be solved is the fabrics wrinkle flu, clothes drape and the sense of hair flowing;

3) The customized human body template to quickly generate the online ordering system : clothing customized 3D body model generated system; Analog and generate a threedimensional human body simulation, express the customization required human eigenvalue and meet the customers' needs of to getting the true effect of the fitting through a network terminal input parameter values in accordance with the requirements of the customers customized apparel categories. Customized templates quickly generated and customized system; the system automatically generate orders and requirements documents (information) in the format which suited for custom processing enterprises after getting the information of the customers' body and style, then transmitted to the clothing technical processing sector over the network. Also it quickly generate the fitting clothing samples and nesting diagram which applied to the physical characteristics of the customers as a response conditions triggering clothing MTM (clothing tailored) . It is the system connected to a network terminal, the integration between the garment CAD and MTM ,the standardized design for data, and the development of various aspects of the interface that achieve the seamless connectivity customization.

\section{III . The achievement in design and function of the digital clothing E-commerce system}

Realize the function of the digital clothing e-commerce system (see Figure 3) through the combination of clothing engineering and the characteristics of the network of retail, which mainly including virtual exhibitions, digitized custom clothing and online retail E-commerce platform.

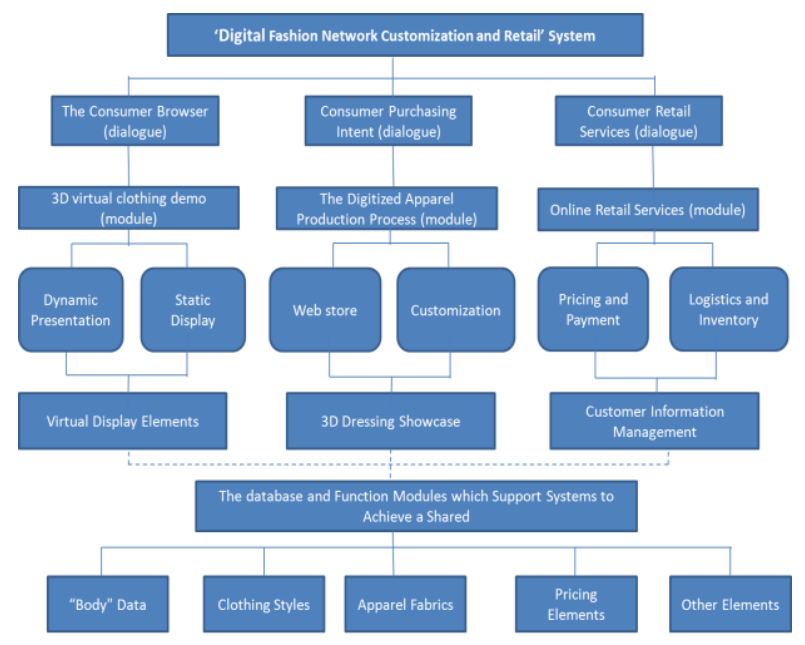

Fig. 3 The digital fashion network customization and the functions of online fitting system

A. The achievement in design and function of the clothing dynamic virtual presentation (show) technology

The key technology of the dynamic clothing demo virtual show platform: human motion simulation system based on the skeleton points and the technology of skinning binding, the establishment of a virtual human motion model database of specific actions, such as regular exercise, catwalk models; fabric simulation system (such as overhanging, elegant, etc.) based particle - spring the mesh, to carry out the correlation research in fabrics basic physical and mechanical properties and fabric simulation, including the grid type, best quality shells coefficient (bending, shear, tensile, other damping ) select, etc. Based on the virtual wearing apparel system with clothing pieces, we can establish a movement model of the fabric (including gravity, virtual suturing force, wearing collision reaction force) and fabric - body collision detection rules (including the way the collision point, line and plane elements intersect with each other, the human body - fabrics and the collision rules for fabrics); establish the fabric motion model includes aerodynamic model (such as a simplified linear/ non-linear lifting force, wind, etc.) based on the virtual wearing apparel system with clothing pieces, the body-fabric motion model (including the mechanical model of fabric mesh for the promotion which body grid surface movement does), to achieve the dynamic display of clothing virtual scene, and 
systematically integrate the background generated by the scene editor with the virtual wearing clothing, as well as specific actions prospect garments dynamic display systems.

\section{B. The customization and the fitting system in the digital online clothing network}

1) The development and function implementation of the fitting technology in clothing online. The key technologies that are involved in the Clothing online 3D virtual to implement the function for fitting platform: the technology for the remote measurement and effective access to the date of human body. Auxiliary measure instructions that are configured in the client-site through e-commerce platform can help customers in the network terminal input human body parameters needed for modeling accurately, with the character description about the size to understand easier, obtaining customer size data. Customer will get the electronic label, store their human body parameter and the size of the appropriate shape; 3D human body reconstruction. Through heterogeneous system integration technology in the foreign country, through the concept of interface, software interface and hardware interface, implement different combinations of hardware and software systems, and integrate "3D human body measurement system", "3D fitting system", and make the data in the smooth transfer and conversion between different systems, and consider the network information and the fitting system of seamless connection, to realize "custom" human body reconstruction;

$3 \mathrm{D}$ virtual dressing. It connects and integrates the thirdparty software, mold design software, 3D body scanning software, and builds 3D virtual fitting system, at the same time to implement article fabrics texture, tone, virtual effect in the system, and to establish design database in the system development; The realization of the online fitting systems; Using B/S mode to realize the network browsing, namely all processing on the server, the client browser is sending and receiving commands; At the same time, in order to reduce the amount of data, to achieve real-time network interaction, this system proceed the optimal interval of image sampling for virtual rendering of the panoramic view of clothing after $3 \mathrm{D}$ reconstruction via the background server, weigh the moderate between in the real-time during the network transmission and the lifelike that $3 \mathrm{D}$ effect shows; The realization of the concept of interface and software interface. According to different system of human body dimension parameters and the definition of different series that is not corresponding to each situation, there will be based on related national standards and international standards, through mathematical modeling, curve fitting and other means, to achieve reunification of the size parameters of the integrated system in human body, the corresponding data interface and fill a vacancy. According to the condition where different system data from different software can't be translated, regards tool software as development platform, depending on the underlying data coding technology, realizes the software interface about the data automatic conversion in the integration system.
2) The technology development and function implementation in the custom clothing network. The key technologies that are involved in the Custom clothing network to implement custom platform function: the establishment of the backstage database; including the size, design, plate type libraries, fabrics library, details library. Realize the individual body shape matching and custody; Custom clothing customization 3D human body model generation system. The system through the network terminal input parameter values of face and form that system specified, it also can be connected to the terminal measurement equipment, import the measured value, the system will automatically identifying customer size, custom-made clothing type according to the customer request, simulating to generate 3D human body, the simulation of the simulated human body has a custom sense, includes all express custom required characteristic values of the human body ,at the same time with vivid visual images to show the stereo effect and meet the needs of customers to obtain the fitting effect, it is a breakthrough currently in the network customization technology, the system Equipped with auxiliary measurement instruction can help customers in any network terminal input parameters required for modeling form accurately; Custom template generation system quickly. After get customer form, design information, the system automatically generate orders to meet the requirements of custom processing enterprises format file, this file that contains the information can be transferred over the network to clothing technology processing department, and as a condition of response that is triggered (customized) clothing, clothing MTM quickly generated form-fitting clothing plate and discharge diagram that meet customer shape characteristics.

\section{The applications of the clothing, digital network business system}

According to Chinese customs statistics, 2012 textile (group) co., LTD. Shanghai textile clothing exports reached $\$ 2.74$ billion, ranks first in export textile and garment industry enterprises in the country. Shanghai textile (group) co., LTD. top the list of "2012 Shanghai enterprise group summary enterprise of tax of 37 in 2012. But the Shanghai textile (group) co., LTD. Electronic business accounts for only 2-5\%. To conform to the group's "twelfth five-year" plan: in order to further implement the "science and technology and fashion" development strategy, focus on building "E-commerce" textile and apparel (including online personalized design and customization) in 2015 and expecting to plan E-commerce in trade volume ratio reached $30 \%$, and become Shanghai, Yangtze river delta region's textile and garment industry chain of E-commerce trade marketing platform.

This project combined with digital creative clothing design, production and retail system, in the modern garment industry shows its unique advantages, which can realize high efficient and fast digital clothing production chain, as a kind of brand-new network clothing digital production and marketing methods, it become a focus point in the study of domestic and foreign clothing production and marketing, and will become an 
important development direction in the future digital garment production. So the research and development of the project is of great practical significance and application value to the construction of Shanghai public service platform, to reform the marketing mode of textile and garment industry chain Ecommerce on traditional garment industry, and to improve level and the enhancement enterprise market competitiveness.

\section{Acknowledgements}

This study was funded by Science Technology Commission of Shanghai Municipality, the funded project No. 11510501600. This study was funded by Municipal Education Commission of Shanghai, the funded project No. 13ZS128

\section{References:}

[1] Shanghai textile group in 2012 the national textile and garment export industry ranked first http://www.shangtex.biz

[2] Shanghai textile group RongLie enterprise group summary tax section 37 http://www.shangtex.biz

[3] Shanghai textile "legend in one hundred year" http://www.shangtex.biz 